\title{
EVOLUTION OF FERMIONIC SYSTEMS AS AN EXPECTATION OVER POISSON PROCESSES
}

\author{
M. BECCARIA \\ Dipartimento di Fisica, Università di Lecce, Via Arnesano, 73100 Lecce, Italy \\ C. PRESILLA \\ Dipartimento di Fisica, Università di Roma "La Sapienza", Piazzale A. Moro 2, 00185 \\ Roma, Italy \\ G. F. DE ANGELIS \\ Dipartimento di Fisica, Università di Lecce, Via Arnesano, 73100 Lecce, Italy \\ G. JONA-LASINIO \\ Dipartimento di Fisica, Università di Roma "La Sapienza", Piazzale A. Moro 2, 00185 \\ Roma, Italy

\begin{abstract}
We derive an exact probabilistic representation for the evolution of a Hubbard model with site- and spin-dependent hopping coefficients and site-dependent interactions in terms of an associated stochastic dynamics of a collection of Poisson
\end{abstract} \\ processes.
}

In a recent papert we provided an exact probabilistic expression for the real time or the imaginary time evolution of a Fermi system, in particular a Hubbard model, in terms of an associated stochastic dynamics of a collection of Poisson processes. Here, we generalize the result to the case of a Hubbard model with site- and spin-dependent hopping coefficients and site-dependent interactions. This situation is of interest if disorder is present 2 .

Let us consider the Hubbard Hamiltonian

$$
H=-\sum_{i=1}^{|\Lambda|} \sum_{j=i}^{|\Lambda|} \sum_{\sigma=\uparrow \downarrow} \eta_{i j \sigma}\left(c_{i \sigma}^{\dagger} c_{j \sigma}+c_{j \sigma}^{\dagger} c_{i \sigma}\right)+\sum_{i=1}^{|\Lambda|} \gamma_{i} c_{i \uparrow}^{\dagger} c_{i \uparrow} c_{i \downarrow}^{\dagger} c_{i \downarrow},
$$

where $\Lambda \subset Z^{d}$ is a finite $d$-dimensional lattice with cardinality $|\Lambda|,\{1, \ldots,|\Lambda|\}$ some total ordering of the lattice points, and $c_{i \sigma}$ the usual anticommuting destruction operators at site $i$ and spin index $\sigma$. Note that the Hamiltonian (11) also allows for spin-dependent site energies $-2 \eta_{i i \sigma}$. We are interested in evaluating the matrix elements $\left\langle\mathbf{n}^{\prime}\left|e^{-i H t}\right| \mathbf{n}\right\rangle$ where $\mathbf{n}=\left(n_{1 \uparrow}, n_{1 \downarrow}, \ldots, n_{|\Lambda| \uparrow}, n_{|\Lambda| \downarrow}\right)$ are the occupation numbers taking the values 0 or 1 . Since the total number of fermions per spin component is a conserved quantity, we consider only configurations $\mathbf{n}$ and $\mathbf{n}^{\prime}$ such that $\sum_{i=1}^{|\Lambda|} n_{i \sigma}^{\prime}=\sum_{i=1}^{|\Lambda|} n_{i \sigma}$ for $\sigma=\uparrow \downarrow$. In the following we shall use the modulus 2 addition $n \oplus n^{\prime}=\left(n+n^{\prime}\right) \bmod 2$.

Let $\Gamma_{\sigma}=\left\{(i, j), 1 \leq i<j \leq|\Lambda|: \eta_{i j \sigma} \neq 0\right\}$ and $\left|\Gamma_{\sigma}\right|$ its cardinality. By introducing

$$
\begin{aligned}
\lambda_{i j \sigma}(\mathbf{n}) & \equiv\left\langle\mathbf{n} \oplus \mathbf{1}_{i \sigma} \oplus \mathbf{1}_{j \sigma}\left|c_{i \sigma}^{\dagger} c_{j \sigma}+c_{j \sigma}^{\dagger} c_{i \sigma}\right| \mathbf{n}\right\rangle \\
& =(-1)^{n_{i \sigma}+\cdots+n_{j-1 \sigma}}\left[n_{j \sigma}\left(n_{i \sigma} \oplus 1\right)-n_{i \sigma}\left(n_{j \sigma} \oplus 1\right)\right],
\end{aligned}
$$


where $\mathbf{1}_{i \sigma}=\left(0, \ldots, 0,1_{i \sigma}, 0, \ldots, 0\right)$, and

$$
V(\mathbf{n}) \equiv\langle\mathbf{n}|H| \mathbf{n}\rangle=\sum_{i=1}^{|\Lambda|} \gamma_{i} n_{i \uparrow} n_{i \downarrow}-\sum_{i=1}^{|\Lambda|} \sum_{\sigma=\uparrow \downarrow} 2 \eta_{i i \sigma} n_{i \sigma},
$$

the following representation holds

$$
\begin{gathered}
\left\langle\mathbf{n}^{\prime}\left|e^{-i H t}\right| \mathbf{n}\right\rangle=\mathbf{E}\left(\delta_{\mathbf{n}^{\prime}, \mathbf{n} \oplus \mathbf{N}^{t}} \mathcal{M}^{t}\right) \\
\mathcal{M}^{t}=\exp \left\{\sum_{\sigma=\uparrow \downarrow} \sum_{(i, j) \in \Gamma_{\sigma}} \int_{[0, t)} \log \left[i \eta_{i j \sigma} \rho_{i j \sigma}^{-1} \lambda_{i j \sigma}\left(\mathbf{n} \oplus \mathbf{N}^{s}\right)\right] d N_{i j \sigma}^{s}\right. \\
\left.-i \int_{0}^{t} V\left(\mathbf{n} \oplus \mathbf{N}^{s}\right) d s+\sum_{\sigma=\uparrow \downarrow} \sum_{(i, j) \in \Gamma_{\sigma}} \rho_{i j \sigma} t\right\} .
\end{gathered}
$$

Here, $\left\{N_{i j \sigma}^{t}\right\},(i, j) \in \Gamma_{\sigma}$ and $\sigma=\uparrow \downarrow$, is a family of $\left|\Gamma_{\uparrow}\right|+\left|\Gamma_{\downarrow}\right|$ independent Poisson processes with parameters $\rho_{i j \sigma}$ and $\mathbf{N}^{t}=\left(N_{1 \uparrow}^{t}, N_{1 \downarrow}^{t}, \ldots, N_{|\Lambda| \uparrow}^{t}, N_{|\Lambda| \downarrow}^{t}\right)$ are $2|\Lambda|$ stochastic processes defined as

$$
N_{k \sigma}^{t}=\sum_{(i, j) \in \Gamma_{\sigma}: i=k \text { or } j=k} N_{i j \sigma}^{t}
$$

We remind that a Poisson process $N^{t}$ with parameter $\rho$ is a jump process characterized by the probabilities $P\left(N^{t+s}-N^{t}=k\right)=(\rho s)^{k} e^{-\rho s} / k$ !. Its trajectories are piecewise-constant increasing integer-valued functions continuous from the left. The stochastic integral $\int d N^{t}$ is just an ordinary Stieltjes integral

$$
\int_{[0, t)} f\left(s, N^{s}\right) d N^{s}=\sum_{k: s_{k}<t} f\left(s_{k}, N^{s_{k}}\right),
$$

where $s_{k}$ are random jump times having probability density $p(s)=\rho e^{-\rho s}$. Finally, the symbol $\mathbf{E}(\ldots)$ is the expectation of the stochastic functional within braces.

The matrix elements $\left\langle\mathbf{n}^{\prime}\left|e^{-i H t}\right| \mathbf{n}\right\rangle$ obey the ODE system

$$
\frac{d}{d t}\left\langle\mathbf{n}^{\prime}\left|e^{-i H t}\right| \mathbf{n}\right\rangle=-i \sum_{\mathbf{n}^{\prime \prime}}\left\langle\mathbf{n}^{\prime}|H| \mathbf{n}^{\prime \prime}\right\rangle\left\langle\mathbf{n}^{\prime \prime}\left|e^{-i H t}\right| \mathbf{n}\right\rangle
$$

with initial condition $\left.\left\langle\mathbf{n}^{\prime}\left|e^{-i H t}\right| \mathbf{n}\right\rangle\right|_{t=0}=\delta_{\mathbf{n}^{\prime} \mathbf{n}}$. One may check that (何) is indeed solution of (7) by applying the rules of stochastic differentiation. We have

$$
\begin{aligned}
\mathbf{E} & \left(\delta_{\mathbf{n}^{\prime}, \mathbf{n} \oplus \mathbf{N}^{t+d t}} \mathcal{M}^{t+d t}\right) \\
= & \sum_{\mathbf{n}^{\prime \prime}} \mathbf{E}\left(\prod_{\sigma=\uparrow \downarrow} \prod_{(i, j) \in \Gamma_{\sigma}} \delta_{\mathbf{n}^{\prime}, \mathbf{n}^{\prime \prime} \oplus d \mathbf{N}^{t}} e^{\int_{[t, t+d t)} \log \left[i \eta_{i j \sigma} \rho_{i j \sigma}^{-1} \lambda_{i j \sigma}\left(\mathbf{n} \oplus \mathbf{N}^{s}\right)\right] d N_{i j \sigma}^{s}}\right. \\
& \left.\times e^{-i V\left(\mathbf{n} \oplus \mathbf{N}^{t}\right) d t+\sum_{\sigma=\uparrow \downarrow} \sum_{(i, j) \in \Gamma_{\sigma}} \rho_{i j \sigma} d t} \delta_{\mathbf{n}^{\prime \prime}, \mathbf{n} \oplus \mathbf{N}^{t}} \mathcal{M}^{t}\right)
\end{aligned}
$$

For the Markov property, the expectation of the factors containing the stochastic integrals in the interval $[t, t+d t]$ can be taken separately. By expanding each one 
of them over all possible numbers of jumps of the Poisson processes as

$$
\begin{aligned}
& \mathbf{E}\left(\delta_{\mathbf{n}^{\prime}, \mathbf{n}^{\prime \prime} \oplus d \mathbf{N}^{t}} e^{\int_{[t, t+d t)} \log \left[i \eta_{i j \sigma} \rho_{i j \sigma}^{-1} \lambda_{i j \sigma}\left(\mathbf{n} \oplus \mathbf{N}^{s}\right)\right] d N_{i j \sigma}^{s}}\right) \\
& =\delta_{\mathbf{n}^{\prime}, \mathbf{n}^{\prime \prime}} e^{0} e^{-\rho_{i j \sigma} d t}+\delta_{\mathbf{n}^{\prime}, \mathbf{n}^{\prime \prime} \oplus \mathbf{1}_{i \sigma} \oplus \mathbf{1}_{j \sigma}} e^{\log \left[i \eta_{i j \sigma} \rho_{i j \sigma}^{-1} \lambda_{i j \sigma}\left(\mathbf{n} \oplus \mathbf{N}^{t}\right)\right]} e^{-\rho_{i j \sigma} d t} \rho_{i j \sigma} d t+\ldots
\end{aligned}
$$

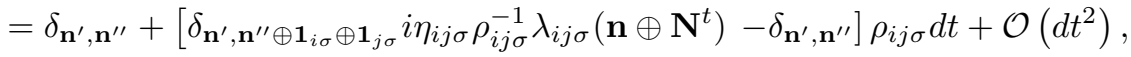

to order $d t$ we obtain

$$
\begin{aligned}
\mathbf{E}\left(\delta_{\mathbf{n}^{\prime}, \mathbf{n} \oplus \mathbf{N}^{t+d t}} \mathcal{M}^{t+d t}\right)= & \sum_{\mathbf{n}^{\prime \prime}}\left[\delta_{\mathbf{n}^{\prime}, \mathbf{n}^{\prime \prime}}+\sum_{\sigma=\uparrow \downarrow} \sum_{(i, j) \in \Gamma_{\sigma}} \delta_{\mathbf{n}^{\prime}, \mathbf{n}^{\prime \prime} \oplus \mathbf{1}_{i \sigma} \oplus \mathbf{1}_{j \sigma}} i \eta_{i j \sigma} \lambda_{i j \sigma}\left(\mathbf{n}^{\prime \prime}\right) d t\right. \\
& \left.-\delta_{\mathbf{n}^{\prime}, \mathbf{n}^{\prime \prime}} i V\left(\mathbf{n}^{\prime \prime}\right) d t\right] \mathbf{E}\left(\delta_{\mathbf{n}^{\prime \prime}, \mathbf{n} \oplus \mathbf{N}^{t}} \mathcal{M}^{t}\right)
\end{aligned}
$$

Finally, we rewrite this relation as

$$
\begin{aligned}
d \mathbf{E}\left(\delta_{\mathbf{n}^{\prime}, \mathbf{n} \oplus \mathbf{N}^{t}} \mathcal{M}^{t}\right) & =\mathbf{E}\left(\delta_{\mathbf{n}^{\prime}, \mathbf{n} \oplus \mathbf{N}^{t+d t}} \mathcal{M}^{t+d t}\right)-\mathbf{E}\left(\delta_{\mathbf{n}^{\prime}, \mathbf{n} \oplus \mathbf{N}^{t}} \mathcal{M}^{t}\right) \\
& =-i \sum_{\mathbf{n}^{\prime \prime}}\left\langle\mathbf{n}^{\prime}|H| \mathbf{n}^{\prime \prime}\right\rangle \mathbf{E}\left(\delta_{\mathbf{n}^{\prime \prime}, \mathbf{n} \oplus \mathbf{N}^{t}} \mathcal{M}^{t}\right) d t
\end{aligned}
$$

It is clear that the fermionic nature of $H$ plays no special role in the above derivation which holds for any system described by a finite Hamiltonian matrix.

In order to construct an efficient algorithm for evaluating (45), we start by observing that the functions $\lambda_{i j \sigma}\left(\mathbf{n} \oplus \mathbf{N}^{s}\right)$ vanish when the occupation numbers $n_{i \sigma} \oplus N_{i \sigma}^{s}$ and $n_{j \sigma} \oplus N_{j \sigma}^{s}$ are equal. We say that for a given value of $\sigma$ the link $i j$ is active at time $s$ if $\lambda_{i j \sigma}\left(\mathbf{n} \oplus \mathbf{N}^{s}\right) \neq 0$. We shall see in a moment that only active links are relevant. Let us consider how the stochastic integral in (5) builds up along a trajectory defined by considering the time ordered succession of jumps in the family $\left\{N_{i j \sigma}^{t}\right\}$. The contribution to the stochastic integral in the exponent of (5) at the time of the first jump, for definiteness suppose that the link $i_{1} j_{1}$ with spin component $\sigma_{1}$ jumps first at time $s_{1}$, is

$$
\log \left[i \eta_{i_{1} j_{1} \sigma_{1}} \rho_{i_{1} j_{1} \sigma_{1}}^{-1} \lambda_{i_{1} j_{1} \sigma_{1}}\left(\mathbf{n} \oplus \mathbf{N}^{s_{1}}\right)\right] \theta\left(t-s_{1}\right)
$$

where $\mathbf{N}^{s_{1}}=\mathbf{0}$ due the assumed left continuity. Therefore, we obtain a real finite or $-\infty$ contribution to the stochastic integral depending if the link $i_{1} j_{1} \sigma_{1}$ was active or not at time 0 . If $s_{1} \geq t$ we have no contribution to the stochastic integral from this trajectory. If $s_{1}<t$ a second jump of a link, suppose $i_{2} j_{2}$ with spin component $\sigma_{2}$, can take place at time $s_{2}>s_{1}$. The analysis can be repeated by considering an arbitrary number of jumps. Of course, when the real part of the stochastic integral is $-\infty$, i.e. when some $\lambda=0$, there is no contribution to the expectation. The other integral in (5) is an ordinary integral of a piecewise constant bounded function.

From the above remarks it is clear that the only trajectories to be considered are those associated to the jumps of active links. We start by determining the set of active links $A_{\sigma}^{1} \subset \Gamma_{\sigma}, \sigma=\uparrow \downarrow$, in the initial configuration $\mathbf{n}$ assigned at time 0 . This is done by inspecting the occupation numbers of the sites according to the rule that the link $i j$ is active for the spin component $\sigma$ if $n_{i \sigma}+n_{j \sigma}=1$. Then, we extract the jump time $s_{1}$ according to the probability density

$$
p(s)=\sum_{\sigma=\uparrow \downarrow} \sum_{(i, j) \in A_{\sigma}^{1}} \rho_{i j \sigma} e^{-\sum_{\sigma=\uparrow \downarrow} \sum_{(i, j) \in A_{\sigma}^{1}} \rho_{i j \sigma} s} .
$$


Finally, we choose the jumping link in the set $A_{\sigma}^{1}$ with probability

$$
P_{i j \sigma}=\frac{\rho_{i j \sigma}}{\sum_{\sigma=\uparrow \downarrow} \sum_{(i, j) \in A_{\sigma}^{1}} \rho_{i j \sigma}} .
$$

The contribution to $\mathcal{M}^{t}$ at the time of the first jump is therefore, up to the last factor which appears in (5),

$$
\begin{aligned}
& i \eta_{i_{1} j_{1} \sigma_{1}} \rho_{i_{1} j_{1} \sigma_{1}}^{-1} \lambda_{i_{1} j_{1} \sigma_{1}}\left(\mathbf{n} \oplus \mathbf{N}^{s_{1}}\right) e^{-i V\left(\mathbf{n} \oplus \mathbf{N}^{s_{1}}\right) s_{1}} e^{-\sum_{\sigma=\uparrow \downarrow} \sum_{(i, j) \in \Gamma_{\sigma} \backslash A_{\sigma}^{1}} \rho_{i j \sigma} s_{1}} \theta\left(t-s_{1}\right) \\
& +e^{-i V\left(\mathbf{n} \oplus \mathbf{N}^{t}\right) t} e^{-\sum_{\sigma=\uparrow \downarrow} \sum_{(i, j) \in \Gamma_{\sigma} \backslash A_{\sigma}^{1}} \rho_{i j \sigma} t} \theta\left(s_{1}-t\right),
\end{aligned}
$$

where $\exp \left(-\sum_{\sigma=\uparrow \downarrow} \sum_{(i, j) \in \Gamma_{\sigma} \backslash A_{\sigma}^{1}} \rho_{i j \sigma} s\right)$ is the probability that the non active links do not jump in the time interval $s$. The contribution of a given trajectory is obtained by multiplying the factors corresponding to the different jumps until the last jump takes place later than $t$. For a given trajectory we thus have

$$
\begin{aligned}
\mathcal{M}^{t}= & \prod_{k \geq 1}\left[i \eta_{i_{k} j_{k} \sigma_{k}} \rho_{i_{k} j_{k} \sigma_{k}}^{-1} \lambda_{i_{k} j_{k} \sigma_{k}}\left(\mathbf{n} \oplus \mathbf{N}^{s_{k}}\right) e^{\left[\zeta_{k}-i V\left(\mathbf{n} \oplus \mathbf{N}^{s_{k}}\right)\right]\left(s_{k}-s_{k-1}\right)} \theta\left(t-s_{k}\right)\right. \\
& \left.+e^{\left[\zeta_{k}-i V\left(\mathbf{n} \oplus \mathbf{N}^{t}\right)\right]\left(t-s_{k-1}\right)} \theta\left(s_{k}-t\right)\right] .
\end{aligned}
$$

Here, $\zeta_{k}=\sum_{\sigma=\uparrow \downarrow} \sum_{(i, j) \in A_{\sigma}^{k}} \rho_{i j \sigma}$ where $A_{\sigma}^{k}$ is the set of active links with spin $\sigma$ in the interval $\left(s_{k-1}, s_{k}\right]$ and $s_{0}=0$. Note that the last exponentially increasing factor in (5) cancels out in the final expression of $\mathcal{M}^{t}$. The analogous expression of $\mathcal{M}^{t}$ for imaginary times is simply obtained by replacing $\eta \rightarrow-i \eta$ and $\gamma \rightarrow-i \gamma$.

In principle, the algorithms parametrized by different $\rho_{i j \sigma}$ are all equivalent as (45) holds for any choice of the Poisson rates. However, since we estimate numerically the expectation values with a finite number of trajectories, this may introduce a systematic error. It can be shown that the best performance is obtained for the natural choice $\rho_{i j \sigma} \sim \eta_{i j \sigma}$ independently of the interaction strength $\gamma$.

\section{References}

1. M. Beccaria, C. Presilla, G. F. De Angelis, and G. Jona-Lasinio, Europhys. Lett. 48, 243 (1999).

2. See, for example, A. W. Sandvik, D. J. Scalapino, and P. Henelius, Phys. Rev. B 50, 10474 (1994). 